\title{
Optimizing Fetal Heart Screening at the Anatomy Ultrasound
}

\section{Dena Towner}

\begin{abstract}
Congenital cardiac abnormalities are one of the most common birth defects at 1 in 120 births. The majority of which have no underlying risk factors. The fetal heart assessment is felt to be the most challenging part of the anatomy examination and most studies report that nearly half of major heart defects are missed, thus, confirming the challenge for antenatal detection rate. This article reviews the key views and key items to assess in fetal heart imaging during the anatomy ultrasound.
\end{abstract}

Keywords: Fetal echo, Fetal heart, Heart defect, Screening.

How to cite this article: Towner D. Optimizing Fetal Heart Screening at the Anatomy Ultrasound. Donald School J Ultrasound Obstet Gynecol 2016;10(1):50-54.

\section{Source of support: Nil}

Conflict of interest: None

\section{INTRODUCTION}

Cardiac abnormalities are the most common birth defects present in 1 of every 120 births. Approximately half of these are major defects, which may require special care or surgery after delivery. Infants with a ductal dependent lesion decompensate rapidly when the ductus closes, which could be after discharge home, leading to serious neurologic injury or death. Likewise, neonates with transposition of the great vessels without a left to right shunt will succumb rapidly without an emergent septostomy. In neonates with these critical lesions, the chance of death before cardiac surgery is reduced $70 \%$ with prenatal diagnosis and planned delivery at a facility capable of neonatal management. ${ }^{1}$ Thus, it is important to identify these babies before birth so that delivery takes place in a facility able to care for the baby to optimize neonatal outcome as well as minimize social and emotional consequences in the family when a neonatal transport is necessary. In this meta-analysis by Holland that demonstrated the improved outcome for

\section{Professor}

Department of Obstetrics and Gynecology and Women's Health John A Burns School of Medicine, University of Hawaii, Honolulu Hawaii, USA

Corresponding Author: Dena Towner, Professor, Department of Obstetrics and Gynecology and Women's Health, 1319 Punahou Street, Suite 824, Honolulu, Hawaii, USA, Phone: 808 203-6500 e-mail: dtowner@hawaii.edu these neonates with critical cardiac lesion, there were over 1300 such neonates, but only 297 of these neonates were diagnosed prenatally.

\section{Screening for Congenital Heart Defects}

Ideally, there would be a screening test that will optimally identify these fetuses; however, there is no simple blood test and the majority $(80-90 \%)$ of fetuses do not have an underlying known risk factor. ${ }^{2,3}$ Factors known to increase the risk for a congenital heart defect (CHD) include pregestational diabetes, maternal phenylketonuria, maternal obesity, first degree relative with a CHD, teratogens, a large nuchal translucency $>3.5 \mathrm{~mm}$, and other structural anomalies or chromosomal abnormality. ${ }^{4-9}$ The positive predictive value for any of these factors is at most 5\% for a fetal heart defect. Guy's hospital in London has reported that of congenital heart defects identified in their unit, $80 \%$ are referred due to suspicion of a heart defect on a routine anatomy scan. ${ }^{3}$

Thus, the routine anatomical survey is the best screening tool to identify if a fetus has a CHD for the 80 to $90 \%$ of fetuses not thought to be at increased risk. However, the heart is technically the most challenging structure to investigate with the screening anatomy scan due to the moving nature of the fetus and heart in addition to the anatomical complexity of the heart. An abnormal four chamber view alone will identify on average $50 \%$ of $\mathrm{CHD}$ cases and adding evaluation of the great vessels can improve the detection rate to $65 \% .^{10}$ This still leaves 40 to $50 \%$ of critical heart defects not identified prenatally, which is not satisfactory. There are four chambers and three levels of connections (systemic veins to atria, atria to ventricles, ventricles to outflow arteries) to assess, thus, why a few single still images of the fetal heart may not demonstrate a significant abnormality. Functional abnormalities likewise cannot be captured in a still image. Dynamic review with either real time or cine-clip imaging is better able to demonstrate fetal heart relationships for the practitioner interpreting the images if they are not the one acquiring the images. The practitioner needs to understand these anatomical relationships to fully be able to interpret images of the fetal heart. There are standard views that should be assessed for a complete fetal heart evaluation and the practitioner should be aware of variations and pitfalls related to theses views. 
To identify that there is a fetal heart defect, it is paramount to recognize that something just does not look the way it is supposed to be. Thus, pattern recognition of what is normal is important to develop. Good equipment and optimization of the equipment is beneficial; however, it is the understanding of what is normal, the ability to recognize the spatial relations of cardiac structures and the skill to image the fetal heart, i.e. key to assessing the fetal heart. The goal of this article is to review the normal anatomical relationships and point out key findings at each view which if not normal would warrant a referral for a formal fetal ECHO.

\section{Fetal Cardiac Examination}

There are 5 key transverse and 3 sagittal views of the fetus required to optimally screen for heart defect. In each view, documenting only static images for interpretation is not as optimal as real time viewing or video clips to assess the dynamic relationships of the structures. In each view, there are several items to be assessed for normalcy that will be reviewed here. If the view is not normal, or one has to force a normal appearing view, this is a clue that something might be wrong. There are two parts to assessing the fetal heart, structure and function. The heart is a pump and development is based on flow through it and blood will flow in the path of least resistance. Thus, disproportion of size in structures can be a clue there is an abnormality in addition to outright structural abnormalities.

\section{Upper Abdomen View}

The first step in assessing the fetal heart is to assess situs. When measuring the abdominal circumference, the abdominal situs is determined by where the stomach is located. The stomach should not just be assumed to be on the left side, one has to confirm it is left sided. The spleen is immediately posterior to the stomach. If the stomach appears to be touching the ribs posterior laterally, then asplenia may be present which is seen in the heterotaxy syndromes. Additionally, in the abdominal circumference view the descending aorta should be just anterior and slightly to the left of the vertebral body, where as the inferior cava can be seen slightly more anterior and to the right of midline. If both these vessels are on the same side of the midline, something is wrong and a referral should made for further investigation.

\section{Four Chamber View}

Moving cephalad from the abdominal circumference view, immediately above the diaphragm in the transverse plane is the four-chamber view. In the fetus, the right and left sides of the heart are similar in size as the right ventricle is the systemic pump in fetal life. The left ventricle is apex forming as in the adult heart. The only chamber mostly in the right side of the chest will be the right atrium. The other three chambers will be mostly to the left of the midline. This view can be obtained in the long axis with the apex of the heart either directly anterior or posterior pointing. In addition, an orthogonal view or subcostal view should be obtained where the ultrasound beam is nearly perpendicular to the septum. In the long axis view, there can be echo dropout creating a false septal defect, whereas a more perpendicular view is better to image for septal defects. If a ventricular septal defect is present, it should be seen in both these views. Use of color flow Doppler is often needed to identify small defects.

In the four-chamber view (Fig. 1), we apply the PASSSSS pneumonic:

Position: The heart should be in the left chest with the anatomical right ventricle anteriorly, the right atrium in the right chest, and the left atrium next to the descending aorta. There should be no vessel or soft tissue between the left atrium and the descending aorta.

Axis: An imaginary line drawn down the septum should angle $45^{\circ}\left( \pm 20^{\circ}\right)$ from the anterior to posterior midline. Septum: The ventricular and atrial septums are assessed in this view for intactness. There is an offset at the level of the mitral and tricuspid valves with the tricuspid valve slightly displaced into the ventricle. This small segment of septum between the AV valves is atrial on the right side facing the right atrium and ventricular on the left facing the ventricular chamber, thus, this small segment is the true atrioventricular septum. At the atrial level, the foramen flap should be seen moving into the left atrium indicating flow of blood from right to left atrium. Size: The heart should visually fill one-third of the chest. The heart circumference to chest circumference ratio is $50 \pm 5 \%$. ${ }^{11}$ When the heart appears large or small, measurements should be taken and compared to growth

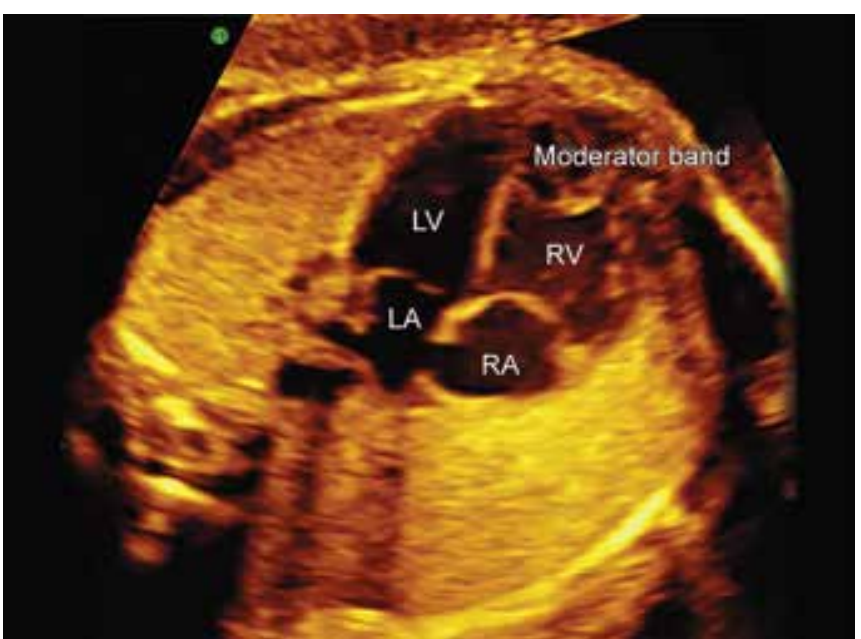

Fig. 1: Four chamber view 
curves to determine if it is the chest or the heart, i.e. potentially abnormal. A skeletal dysplasia with short ribs can lead to a large appearing heart, but it is really a small chest. If the chest is normal size and the heart is large, this is suggestive of a cardiomyopathy developing or a high output cardiac state.

Situs: The apex of the heart should point to the left. Merely determining if the heart is pointing in the correct direction is not all that should be assessed. Determining anatomical correctness of the chambers should also be performed. To confirm the anterior ventricle is the morphologic right ventricle, it should contain the moderator band and the tricuspid valve is the more apically placed valve with a septal leaflet and papillary muscle. Likewise assessment of the atrial chambers should note the anterior chamber being rounded consistent with this being the right atria. The posterior left atria will have irregular walls (pulmonary veins entering) and the foramen flap moving into the atria.

Symmetry: Are the ventricles the same size and similar thickness?

Squeeze: Regular rate noted and normal contractility. Atrial rate is the same as ventricular rate. Fractional shortening measured by M-mode should be $>25 \%$.

\section{Left Ventricular Outflow Tract}

Angling cephalad from the four-chamber view takes one to the left ventricular outflow tract. The root of the aorta comes off the left ventricle centrally in the chest and aims toward the right. Key items to take note of in this view (Fig. 2) include the continuity of the ventricular septum with the anterior aortic wall, the aortic valve opens completely and the leaflets disappear in systole, and the aorta does not bifurcate. The anterior mitral valve leaflet is in continuity with the posterior wall of the aorta. If a Ventricular septal defect (VSD) is present in the perimembranous area of the septum, there will be

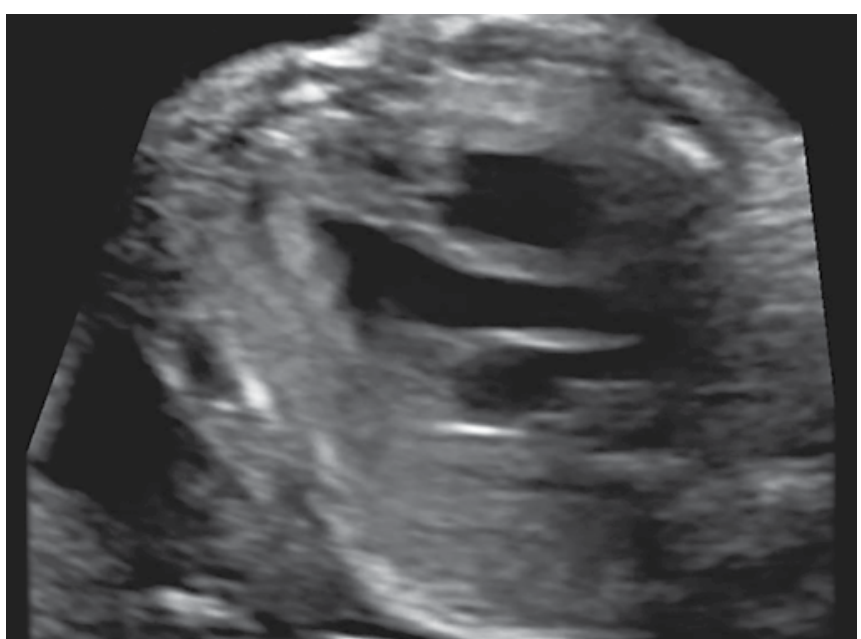

Fig. 2: Left ventricular outflow tract view discontinuity or even the appearance of an over-riding aorta in this view. By noting the aortic valve opening widely to the point of the leaflets disappearing, it is unlikely there is aortic valve stenosis. Likewise, observing past the aortic root for evidence for bifurcation, one can assess for transposition of the great vessels in this view.

\section{Right Outflow Tract View}

Moving slightly cephalad from the left outflow tract view, one will find the right outflow tract (Fig. 3) where the main pulmonary artery (MPA) originates near the anterior chest wall, slightly more cranially than the aorta, and goes toward the spine. The MPA trifurcates shortly after the origination with the right branch pulmonary artery going posterior to the aorta to the right lung, the left branch PA going slightly inferior out of the plane toward the left lung, and the ductus arteriosus continuing on to the descending aorta. The right heart structures surround the aortic root at this level with the right atria and tricuspid valve to the right, the right ventricle more anteriorly, with the pulmonary valve and MPA to the left. Key items to note in this view include the branching of the pulmonary artery and the same size of the aorta and main pulmonary artery. Pulmonary outflow VSD's will be present in the short segment of septum between the tricuspid and pulmonary valves in this view.

\section{Three Vessel Trachea View}

Moving more cephalad in the upper mediastinum, one comes to the three-vessel trachea view (Fig. 4). The superior vena cava is the most right-sided vessel as it is going toward the right atrium, the most rightward structure. It is also the smallest and most posterior of the three vessels as it enters the right atrium at the base of the heart. The aorta is the middle vessel as it exited from the center of the heart with the direction of the vessel toward the right. The pulmonary artery/ductus

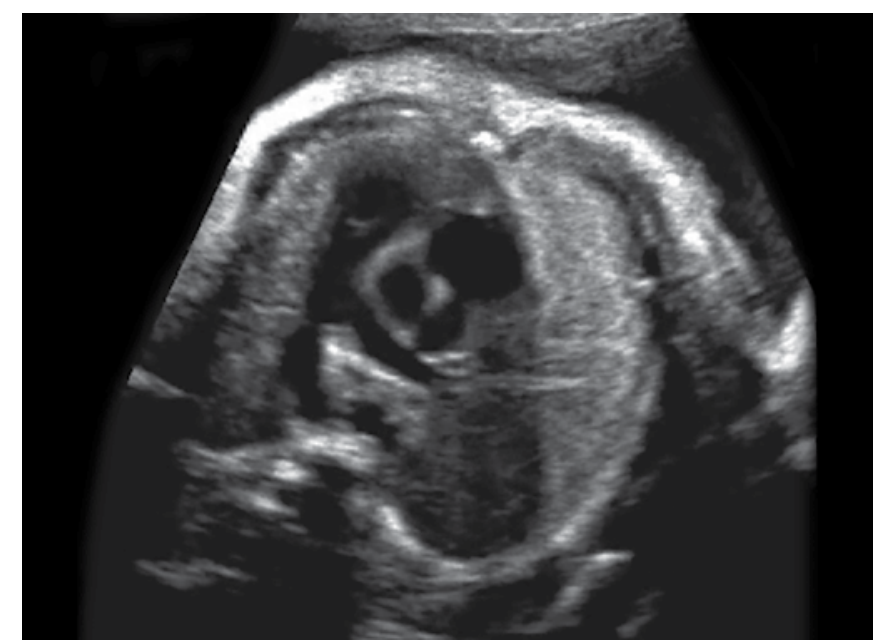

Fig. 3: Right ventricular outflow tract view 


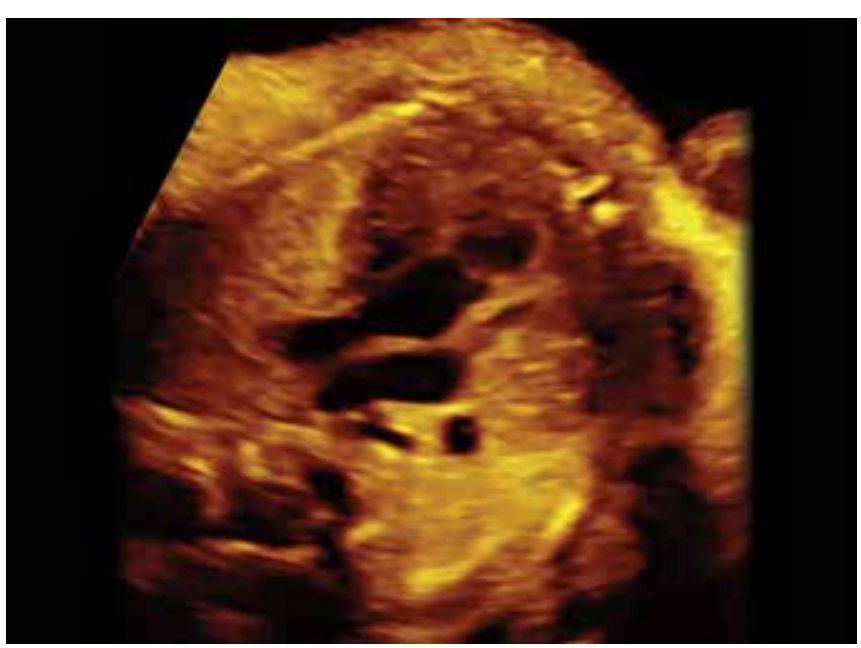

Fig. 4: Three vassel view

arteriosus is the largest and most leftward vessel. As this vessel originates anteriorly from the heart, it will be the most anterior vessel in this view. In this view, the tissue mass anterior to the three vessels is the thymus. The trachea is posterior to the superior vena cava and to the right of the aorta. In this view, you can follow the ductus and aorta to where they join the descending aorta. The aorta at this level should be $>75 \%$ of the ductus in width. With coarctation of the aorta, the aorta will be $<$ $75 \%$ of the size of the ductus. This upper view where the aorta and ductus join has been termed the ' $\mathrm{V}$ view'. The key to the three-vessel trachea view is the order, size, and placement of the three vessels and the trachea. If there are too few or too many vessels or structures that are larger or smaller than expected, there is likely an abnormality underlying the finding and should be referred for further evaluation.

\section{Sagittal Views}

In the sagittal plane, there are three views one should obtain for a complete assessment of the fetal heart. The ductal arch (Fig. 5A) is found in a nearly mid sagittal plane starting anteriorly and directed straight posteriorly to the descending aorta, making a 'hockey stick' appearance to the vessel. The aortic arch (Fig. 5B) is slightly to the fetal right and originates from the middle of the chest and has the head and neck vessels coming off at the top of the arch. The bicaval view (Fig. 5C) is to the right of midline and has the superior and inferior vena cava entering into the right atrium, which creates a 'longhorn bull head' appearance.

\section{Subsequent Ultrasounds}

Many pregnancies have subsequent ultrasounds after the mid-trimester anatomy ultrasound. Cardiac views should again be obtained and reviewed, as there are many lesions
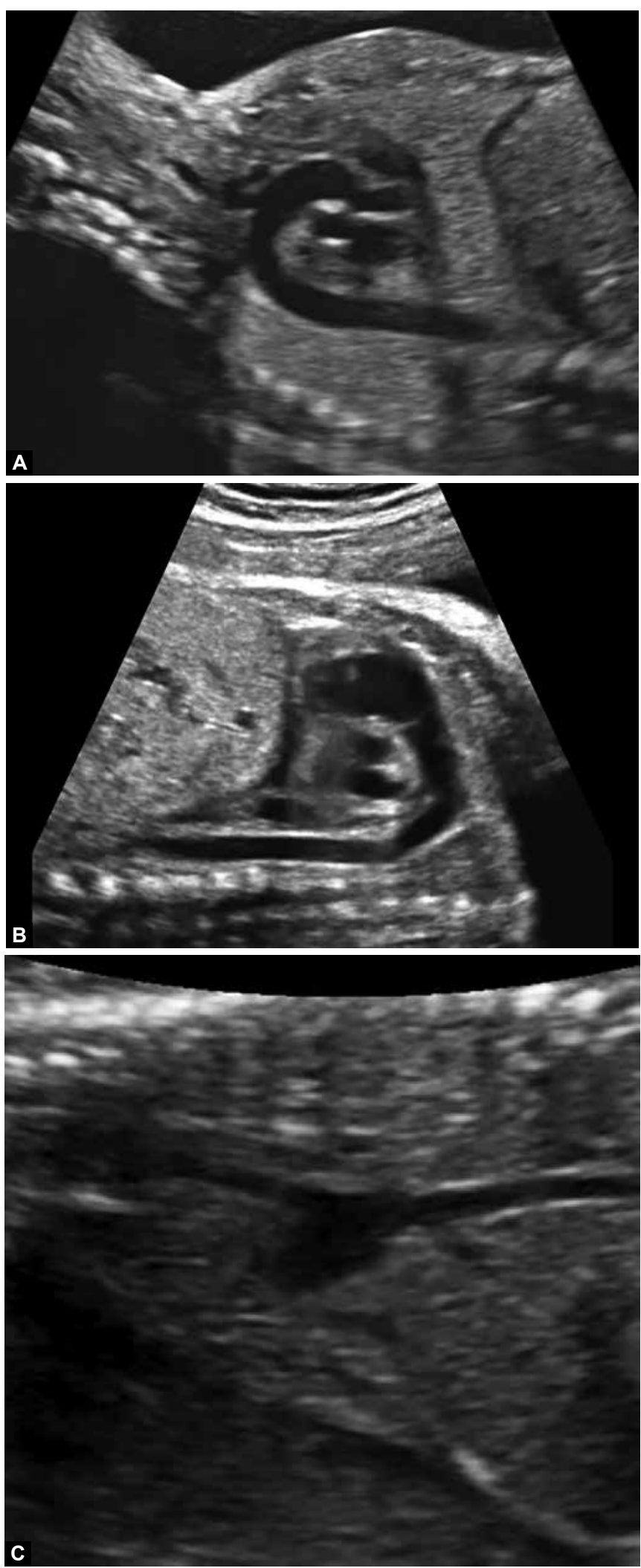

Figs 5A to C: (A) Aortic arch view, (B) ductal arch view and (C) bicaval view

that evolve over gestation or become more evident later in gestation, including valvular stenosis, coarctation, septal defects, endocardial fibroelastosis and rhabdomyomas. Additionally a new issue, such as cardiomyopathy or arrhythmia could have developed. 


\section{CONCLUSION}

The examination of the fetal heart during the screening anatomy ultrasounds is important yet challenging. This review outlined the key components in determining normalcy of the fetal heart. To be comfortable assessing the fetal heart, practitioners of screening anatomy ultrasounds should become familiar with the normal heart anatomy and relationships. Any deviation should alert the practitioner that there may be a problem and further evaluation by providers experienced in fetal echocardiography is warranted. Recognizing the importance of multiple views has improved detection rates from approximately $50 \%$ with the four-chamber view only to approximately $60 \%$ by adding in the great vessels and then to $75 \%$ with adding in the three vessel trachea view. ${ }^{10,12}$ There will be minor heart defects missed, however, it is the major ones that need to be identified prenatally for neonatal benefit in planning site of delivery.

\section{REFERENCES}

1. Holland BJ, Myers JA, Woods CR Jr. Prenatal diagnosis of critical congenital heart disease reduces risk of death from cardiovascular compromise prior to planned neonatal cardiac surgery: a meta-analysis. Ultrasound Obstet Gynecol 2015 Jun;45(6):631-638.

2. Cooper MJ, Enderlein MA, Dyson DC, Roge CL, Tarnoff H. Fetal echocardiography: retrospective review of clinical experience and an evaluation of indications. Obstet Gynecol 1995 Oct;86(4 Pt 1):577-582.
3. Sharland G. Routine fetal cardiac screening: what are we doing and what should we do? Prenatal diagnosis 2004 Dec 30;24(13):1123-1129.

4. Boughman JA, Berg KA, Astemborski JA, et al. Familial risks of congenital heart defect assessed in a population-based epidemiologic study. Am J Med Genet 1987 Apr;26(4):839-849.

5. Hornberger LK. Maternal diabetes and the fetal heart. Heart 2006 Aug;92(8):1019-1021.

6. Jelliffe-Pawlowski LL, Norton ME, Shaw GM, et al. Risk of critical congenital heart defects by nuchal translucency norms. Am J Obstet Gynecol 2015 Apr;212(4):518 e511-510.

7. Loffredo CA. Epidemiology of cardiovascular malformations: prevalence and risk factors. Am J Med Genet 2000 Winter; 97(4):319-325.

8. Rychik J, Ayres N, Cuneo B, et al. American Society of Echocardiography guidelines and standards for performance of the fetal echocardiogram. J Am Soc Echocardiogr 2004 Jul;17(7):803-810.

9. Sotiriadis A, Papatheodorou S, Eleftheriades M, Makrydimas G. Nuchal translucency and major congenital heart defects in fetuses with normal karyotype: a meta-analysis. Ultrasound Obstet Gynecol 2013 Oct;42(4):383-389.

10. Li Y, Hua Y, Fang J, et al. Performance of different scan protocols of fetal echocardiography in the diagnosis of fetal congenital heart disease: a systematic review and metaanalysis. PloS one 2013;8(6):e65484.

11. Paladini D, Chita SK, Allan LD. Prenatal measurement of cardiothoracic ratio in evaluation of heart disease. Arch Dis Child 1990 Jan;65(1 Spec No):20-23.

12. Liu H, Zhou J, Feng QL, et al. Fetal echocardiography for congenital heart disease diagnosis: a meta-analysis, power analysis and missing data analysis. Europ J Prev Cardiol 2014 Sep 25. 